\title{
User involvement in developing mobile and temporarily interconnected systems
}

\author{
Ola Henfridsson* \& Rikard Lindgren ${ }^{\dagger}$ \\ *Viktoria Institute, Hörselgången 4, 41756 Göteborg, Sweden, email: \\ ola.henfridsson@viktoria.se, and †Viktoria Institute, Hörselgången 4, 41756 Göteborg, \\ Sweden, email: rikard.lindgren@viktoria.se
}

\begin{abstract}
Information systems (IS) research on user involvement has primarily theorized relationships between developers, managers and users in systems development. However, so far, marginal attention has been paid to differences in user involvement practices between information systems. This paper explores user involvement in developing mobile and temporarily interconnected systems (MTIS). We refer to MTIS as heterogeneous systems that rely on network technologies for increasing the ubiquity of information services for users on the move. Such systems are becoming increasingly important in leveraging, e.g. car infotainment, supply chain management and wireless e-commerce. With particular emphasis on the nature of MTIS and its implications for user involvement, the paper analyses the systems development process of an action research project. The findings suggest that user involvement practices need to be adapted to accommodate features of this class of systems. Being an early attempt to trace the implications of technology features such as use context switches and temporary system relationships, the paper contributes to the development of an updated theory of the user role in an era of increased system complexity and stakeholder ambiguity.
\end{abstract}

Keywords: heterogeneity, mobile and temporarily interconnected systems, systems development, temporary system relationships, use context switches, user involvement

\section{INTRODUCTION}

User involvement is commonly viewed as a key element in developing information systems that are useful to both host organizations and users. Yet, over the years, such involvement has been contested (Kujala, 2003; Markus \& Mao, 2004; livari, 2006). Critical examinations point to a number of problems including asymmetrical power relations (Howcraft \& Wilson, 2003), conflicts between user groups (Wilson et al., 1997), developers' attitudes and perceptions of their role (Beath \& Orlikowski, 1994), insufficient user motivation, commitment, and incentives (Sabherwal \& Elam, 1995; Cooper, 2000), resource allocation difficulties (Hawk \& Dos Santos, 1991), 
users' lack of technical knowledge (Newman \& Noble, 1990) and user-developer communication (Gallivan \& Keil, 2003). While received theory concentrates on relationships between developers, managers and users, however, implications of changes in the underlying technology for systems development and user involvement are relatively unexplored in the literature.

Network technologies such as Bluetooth and Wireless Local Area Network (WLAN) are increasingly important for interconnecting heterogeneous systems. Relying on network technologies for delivering anytime-anywhere computing (March et al., 2000), mobile and temporarily interconnected systems (MTIS) are the focus of this paper. This class of systems is intended to place the computer backstage and to increase the ubiquity of information services for users on the move (cf. Weiser, 1991). Being part of so-called ubiquitous information environments (Lyytinen \& Yoo, 2002a), MTIS are typically embedded in the physical infrastructure. Such infrastructure may be buildings, containers, goods, roadside infrastructure or as in the case studied in this paper: cars. As will be demonstrated, MTIS are characterized by two properties; they rely on temporary relationships between heterogeneous systems and enable use context switching.

The starting-point of the paper is that the received view of user involvement does not accommodate the nature of systems that support ubiquity of information services. This also goes for more recent user involvement literature on, e.g. wide-audience requirement elicitation (Tuunanen, 2003) and off-the-shelf software (Hansson et al., 2006; Holmström \& Henfridsson, 2006). This is unfortunate given that user involvement is important in developing MTIS.

Focusing on the systems development process of an action research project (see Henfridsson \& Lindgren, 2005), the paper addresses the research question as to how user involvement practices can be adapted to the development of MTIS. The paper contributes to the stream of IS research seeking to build the foundations for an updated theory capable of embracing user involvement in changing IS practice (see e.g. Markus \& Mao, 2004).

\section{THEORETICAL BACKGROUND}

The extant literature lacks a commonly agreed definition of user involvement (Kujala, 2003). Not only do different definitions exist, but user-centred research displays variety with regard to their main locus. Indeed, user-centred dimensions found in the literature include user focus, work-centredness, user participation and personalization (livari \& livari, 2006). These dimensions largely manifest different research traditions.

Barki \& Hartwick's (1989) classic definition portrays user involvement as 'a subjective psychological state reflecting the importance and personal relevance of a system to a user'. However, the definition used throughout this paper reflects the broader view appearing in the literature, where user involvement can be seen as contact with users in different phases of a system development process (cf. Kujala, 2003). Such contact can be either informative, consultative and participative (Damodaran, 1996), indicating the role attributed to users in the project. In product development settings, usability engineers sometimes represent users (Cooper \& Bowers, 1997), serving as surrogate users (livari \& livari, 2006). Ultimately, the goal of user involvement is to assist designers with application-domain knowledge in developing 
systems that are useful to both the host organization and users (Gould \& Lewis, 1985; Wilson et al., 1997).

Early on, Enid Mumford's work at the Tavistock Institute, and in particular the ETHICS methodology (Mumford, 1983), stimulated a wide uptake of user involvement ideas in IS (Mumford, 1993; Hirschheim \& Klein, 1994; Adman \& Warren, 2000). Another stream of inquiry, referred to as the trade-unionist approach (livari \& Lyytinen, 1998), focused on emancipation of resource weak groups in industrial settings (Kyng \& Mathiassen, 1982; Ehn, 1988; Greenbaum \& Kyng, 1991). However, as the field has matured, numerous approaches and methodologies have emerged (Muller et al., 1997; Kujala, 2003). Examples of such approaches and methodologies include contextual design (Beyer \& Holtzblatt, 1995; 1999), co-operative or participatory design (Ehn, 1993), customer-developer links (Keil \& Carmel, 1995), ethnography in systems design (Hughes et al., 1992) and usability engineering (Wixon \& Wilson, 1997). Indeed, it is fair to say that most contemporary approaches to systems development view user involvement to be a natural ingredient (Kujala, 2003; Markus \& Mao, 2004; livari, 2006).

Lately, the IS literature has recognized new forms of user involvement related to the emergence of new technologies. Examples of technology-mediated forms of user involvement are online communities for packaged software development (Holmström \& Henfridsson, 2006) and participation taking place in different forms of open source communities (Fitzgerald, 2006). These user involvement forms are enabled by innovative use of internet technology for stimulating knowledge creation in the development process (Lee \& Cole, 2003). Other forms are related to a new category of end-users associated with off-the-shelf software (Hansson et al., 2006) and wide-audience information systems (Tuunanen, 2003). These users are largely distant and unknown (Grudin, 1991; Sawyer, 2000).

Concurring with the cases of packaged software and wide-audience information systems, systems that support ubiquity of information services involve a distant and unknown user collective. In fact, even the type of computing device on which such services are running is usually unknown. The device can be a cellphone, personal navigation device or BlackBerry, meaning that 'designers of services can only make minimal assumptions about physical computing devices to provide a maximum level of personalization' (Lyytinen \& Yoo, 2002b, p. 380). This is different from traditional systems development where designers can make explicit assumptions about users and their information technology (IT) support.

Few, if any, studies focus on the influences of particular features of software systems on the conditions for accomplishing useful user involvement. Addressing this gap in the literature, this paper explores user involvement in developing MTIS. In what follows, we identify two properties of such systems and discuss these in view of core assumptions in the user involvement literature. We refer to them as switching contexts and temporary relationships between heterogeneous systems.

\section{Use context switches}

MTIS support use patterns established over traditionally bounded use contexts. As noted in the literature, information services should be capable of dynamically recognizing the multifaceted 
context of their use and take appropriate action when it changes (Dey et al., 2001). In this regard, MTIS must simultaneously be designed to provide transparent, integrated and convenient support in localized use contexts (Kleinrock, 2001). Indeed, designing universal services expected to work in varying local contexts is challenging. In the literature, this particular problem has been referred to as multi-contextuality, i.e. the coexistence of different use contexts (Henfridsson \& Lindgren, 2005; Lindgren et al., 2008). While traditional user involvement studies do not necessarily exclude the existence of multiple contexts, they do assume that contexts are non-switching. This means that actions need to be taken for capturing use patterns inherited from other contexts. It is therefore critical to explore consequences of switching contexts for user involvement practices.

\section{Q1}

How should a system designer appropriate, and determine the relevance of, use patterns originating from other contexts when developing MTIS?

\section{Temporary system relationships}

Services in ubiquitous information environments should be capable of seamlessly accessing other computing components to attain the resources necessary for completing the user's task without user manipulation (Abowd \& Mynatt, 2000). In this way, an MTIS must be capable of identifying, adapting and delivering the appropriate combination of computing resources to provide a solution to a user's needs (Andersson \& Lindgren, 2005). However, such temporary system relationships, only functioning when both ends of the system are interoperable, are heterogeneous in that they typically build on IT bases (Lyytinen \& Rose, 2003) with different innovation trajectories. For instance, in being embedded in physical infrastructure such as buildings and cars, MTIS often follow the relatively long life cycles of their hosts, while the connecting device typically follows the consumer electronics industry's short life cycle (Broy et al., 2007). With new connecting devices, there will be new use cases and user expectations, threatening to rapidly erode user value of the MTIS. Thus, it can be assumed important for the designer of such systems to find ways to secure user involvement over time.

\section{Q2}

How can a system designer secure user involvement over time to handle the temporary relationships of MTIS?

As documented in the previous discussion, there is a set of properties of MTIS that breaks with the assumed nature of underlying technologies in the user involvement literature. In this vein, this paper addresses the overall research question as to how user involvement practices can be adapted to the development of MTIS to better cater for dimensions of switching contexts and temporary relationships between heterogeneous systems. 


\section{METHODOLOGY}

This research provides a retrospective account of a grounded action research (Baskerville \& Pries-Heje, 1999; Davison et al., 2004) study reported in Henfridsson \& Lindgren (2005). Conducted in collaboration with Saab Automobile, Mecel and Vodafone, the research followed the same type of design-oriented action research methodology (Lindgren et al., 2004; Andersson et al., 2008) that Cole et al. (2005) describe as a possible instantiation of an integrated approach between action and design research. To this end, a significant element in this action research was the design of an in-car system intended to support convenient and safe use of cellphones. We called this system prototype SeamlessTalk, as to reflect the seamlessness enabled by the system in sustaining phone conversations over use contexts (inside car/outside car).

In this paper, we examine the nature and role of user involvement in the design process of SeamlessTalk. The reason for giving this retrospective account is the belief that MTIS, such as SeamlessTalk, require adapted user involvement practices that partly break with extant assumptions in the literature. While the original study investigated multi-contextuality for contributing to the ubiquitous computing literature (Henfridsson \& Lindgren, 2005), this account focuses on the user involvement lessons learned throughout the project. To enhance the retrospective account, we conducted semi-structured interviews with Saab Automobile employees to follow up on the systems development process.

The action research project covered data collection methods such as interviews, participant observation, technology review and workshop sessions. For the purposes of this paper, we concentrate on the data sources that concern the systems development part of the project. These sources cover the requirement determination and system evaluation phases. The requirements determination phase was conducted for better understanding existing cellphone use in cars. This study involved a study of 18 frequent cellphone users who provided their view on incentives and problems with in-car use of phone use through interviews. The recorded and transcribed interview material worked as a resource in determining high-level requirements that informed the design of SeamlessTalk.

The system evaluation assessed SeamlessTalk in actual use contexts. Six users had SeamlessTalk implemented in their Saab 9-3s and evaluated the prototype for a 2-month period. The six users used the prototype in virtually all car travelling, including everyday commuting, travelling between work and customer sites, weekend trips and so on. They reported problems and general reflections throughout the project but the main sources were an interview study and participant observation. Six recorded and transcribed interviews were conducted at the end of the evaluation. The participant observation primarily concerned system walk-troughs, where users demonstrated highlights and problems with SeamlessTalk that they had encountered during the evaluation phase. The main objective of the evaluation from a systems development point of view was to generate user-based suggestions for improvement of the system.

In addition to these data sources, we conducted an interview study with Saab Automobile employees involved in the systems development process. Covering reflections on user 
involvement in developing SeamlessTalk, the interviews gave insights into the specific nature of adopting user views in developing and launching a networked system. In total, eight semi-structured interviews were conducted with managers and engineers in the Research \& Development (R\&D) and marketing departments.

The analysis of the data material provided a new angle on the phenomenon, motivating a reassessment of the previously conducted Action Research (AR) project. Concurring with the hermeneutic circle in interpretive analysis (Klein \& Myers, 1999), this process involved going back and forth between parts and the whole of the investigated phenomenon. The focus of our project provided a detailed understanding of user requirements and suggestions for MTIS, and, in particular, a view of the particular nature of user involvement in developing such systems. Complemented with the additional interview study, we therefore present a retrospective account of our action research study in order to provide a comprehensive analysis of user involvement in developing MTIS.

\section{USER INVOLVEMENT IN DEVELOPING A NETWORKED SYSTEM}

\section{Development setting}

Traditionally, automakers support in-car communication by embedding a phone module, dial pad and loudspeaker system in the car. Such functionality is sold to end customers as optional choices in top-end car models. However, the rapid customer uptake of mobile devices such cellphones, portable navigation devices and $\mathrm{mp} 3$ players has occasioned a strategy shift. Most automakers currently invest considerable energy in developing in-car systems for supporting personal, mobile devices that are brought into the vehicle. Users can then use their ordinary device in the car and still benefit from a car-integrated hands-free interface. In the automotive industry, this type of systems is usually referred to as nomadic device integration (NDI) or personal telematics systems.

NDI systems are an example of an MTIS. First, the use of NDI systems depends on integrations with technologies that the automaker does not control. Thus, NDI systems need to handle a multitude of devices over time. Designers of NDI systems can therefore only make minimal assumptions about the computing device and service supported. Second, the services supported by an NDI system follow the mobility of their users. This means that the system must be capable of handling users' context switches as to be seamless and convenient. Finally, NDI systems support temporary relationships between heterogeneous systems. They usually build on network technologies such as Bluetooth for establishing ad-hoc connections with mobile devices. In other words, there is no permanent relationship between systems.

In collaboration with Saab Automobile's Advanced Engineering department, the system development process started from the idea that MTIS need to be developed as to match present and future requirements of mobile users. User involvement was important at two distinct phases of the project: requirement determination and system evaluation. In responding to the evaluation of the system developed, Saab also had to consider user requirements 
and preferences concerning the connecting systems of which they had no control: mobile devices.

\section{Determining user requirements}

In 2002, there were no NDI systems on the market. In determining requirements, attention was therefore geared towards existing patterns of cellphone use in cars. Users were thus recruited as representatives for future users. Given that this was a product development setting, the 18 users were surrogate users in the sense that they could be typical users (cf. livari \& livari, 2006). Serving in this role, they were important informants in the process of determining requirements for NDI systems. This user collective, representing different backgrounds but selected for their high level of phone use, was interviewed about their current in-car mobile device practices. Without existing NDI use patterns and a distinct user collective, the interview study became a valuable resource in outlining a design direction. Clearly, there was a time-space discontinuity here that resembles the cases of packaged software, open source and wide-audience information systems.

Our user collective identified a number of use incentives and problems with cellphone use in cars. Indeed, the user views were important inputs in the requirements determination phase. First, the use incentives (meaningful time usage and time-dependant coordination of, e.g. household matters, meeting-times and location) of cellphone use confirmed that it was worth going forward developing a NDI system, which eventually would be evaluated through a more participative mode of user involvement. Second, the results pointed out the problem areas that needed to be addressed in future NDI solutions. The problems regarding mobile device manipulation, call taking, context change and hands-free were all aspects that we thought would be possible to address. We therefore continued by developing design principles that later were instantiated in the SeamlessTalk system. The system and embedded design principles are described below.

\section{SeamlessTalk}

SeamlessTalk was developed and installed as a component of the top-end infotainment system of the Saab 9-3 car. SeamlessTalk is accessible from the main menu of the car infotainment system and establishes a Bluetooth connection between the car (and in-car resources such as dashboard control buttons, dashboard-integrated screen, microphone and audio system) and the cellphone. Even though SeamlessTalk builds on Bluetooth's hands-free profile (HFP), few, if any, mobile devices supported the profile at the time of system implementation. For the purposes of the project, SeamlessTalk was thus developed for the Nokia $6310 i$ and Nokia 6650 phones.

On the basis of our user collective's description of incentives and problems, three system design principles were developed and implemented. First, the service synchronization principle specifies that the NDI system should make selective services associated with the mobile device and deemed plausible for the car setting available to users. Such synchronization was 
achieved by using the Bluetooth ad hoc networking standard with the service discovery protocol (SDP) and the HFP. Users are thus able to use the call service available via the mobile device by selecting the 'hands-free' option available via the user interface of the in-car infotainment system. In this way, the mobile device is not involved at all from a user perspective. This principle corresponded to the insufficient integration problem related to hands-free use, which users pointed out in the requirements determination phase.

Second, the principle of contextually adapted manipulation posits that the user should be provided with device or service controls that are adapted to the spatio-temporal conditions of cars. With SeamlessTalk, users are able to use dashboard controls for controlling the device. The infotainment unit consists of a complete dial pad. Moreover, calls can be executed and terminated by using the yes/no buttons on the steering wheel. Apart from dashboard buttons, the cellphone can also be manipulated by navigating the menus of the infotainment system. This design principle was intended to alleviate the call taking and device manipulation problems reported by the user collective.

Lastly, the context switching support principle specifies that the system should support switching between physical and social contexts, i.e. support seamless transitions across contexts necessary for sustained services. With SeamlessTalk, users can conveniently switch context during ongoing calls. In case of entering the car with an ongoing call, the call can be transferred by using the 'hands-free' option of the infotainment system. Similarly, the call can be transferred to the mobile phone in case of leaving the car. This can also be used in situations where several people are in the car and the SeamlessTalk user wants to make or take a private call, i.e. a social context switch. This principle addresses the context change problem identified in the requirements determination phase.

\section{System evaluation}

SeamlessTalk was evaluated over a 2-month period of authentic use. Five Saab 9-3 cars with SeamlessTalk installed were used. Because two users had the same car, a total of six users appropriated SeamlessTalk in their daily life including commuting, travelling between customer and work sites, leisure trips and so on. The users were asked to reflect upon the impact of the system on their daily mobility and report any technical problems occurring during the evaluation period. Given the duration, authentic use context and intensive developer-user relationship, the users were encouraged to adopt a participative type of involvement (cf. Damodaran, 1996). We thought this type of involvement was required to assess the ubiquity that the system was intended to support.

Over the evaluation, it was noted that service synchronization indeed addressed the handsfree problems identified in the requirements determination phase. Its implementation in the SeamlessTalk system contributed to users' increased convenience and enhanced hands-free use. Similarly, the evaluation showed anticipated results over the two other design principles. The implementation of the contextually adapted manipulation and context-switching principles was encouraging in that SeamlessTalk contributed to improved call taking abilities, facilitated mobile device manipulation, as well as generally convenient context switches. However, the 
evaluation also pinpointed a set of socio-technical issues with far-reaching implications for systems development and user involvement in networked system contexts.

For instance, differences in individual use patterns make contextualized delivery of massscale services difficult. Given the openness of mobile devices, users' appropriation of services in their day-to-day usage can yield an abundance of use patterns. Moreover, these patterns are fluid in that they change over time. The abundance and fluidity of use patterns are problematic aspects for designers of MTIS. In the SeamlessTalk case, it was clear that the lack of synchronization of contact and recent number lists downplayed the convenience of in-car usage for our respondents. One of the users commented:

Today, you never actually dial a number on a mobile phone but you search the contacts you've got. Once you have dialed them, you use the recent number list. That's the routinized behavior once you get seated in the car. Then, you have to search for numbers ... That's a bit stupid. Even more so in the case when the contact list [of the mobile phone] and the application are separated.

The lack of contact list synchronization was commented by several users, indicating its importance in delivering convenient and seamless support. The users thought that such synchronization would be a self-evident feature in a future system. Given SeamlessTalk's interoperability being limited to merely two Nokia phones, support for contact list synchronization would have been straightforward to implement in the prototype. However, the problem for the designers was that this user request could not be addressed in a meaningful way. A general purpose solution must support the plethora of existing and future phone models. Such a solution calls for standard development and adoption, which was beyond the scope of the project.

In addition, the user evaluation highlighted that SeamlessTalk generally supported convenient context-switching. Compliance with the HFP and the SDP generally provided smooth transitions between the in-car context and other contexts. Calls need not be terminated, but could be sustained over different contexts. One observation noted in this regard was that users, at some occasions, were unsure whether or not the hands-free mode was activated:

The context switches are somewhat unpredictable and creates uncertainty. I don't know what it is, how it works . . Consider what happened yesterday: I stopped at the corner shop and switched off the engine. Attempting to avoid disconnecting the phone I left it in the car. When I came back I was still connected, wasn't I? But I don't know, do I?

Needless to say, such uncertainty downplays the perceived user value. We therefore concluded that mode awareness is an important condition for trustful context-switches. From the project's point-of-view, such mode awareness could have easily been implemented in the car end. The mobile device end, however, is out-of-reach for automakers in general and for the project in particular. In other words, despite unambiguous user feedback, the system designers were unable to implement a satisfactory solution because they did not control both ends of the networked system. 


\section{From prototype to software product}

Given the user appreciation that SeamlessTalk rendered, the R\&D department determined to hand over the system concept to the product line organization. However, this decision coincided with a caution about possible problems associated with maintaining user value of a networked system product over time. In particular, NDI solutions rely on connecting devices outside the automaker's control. An infotainment manager at Saab elaborated on this difficulty:

A major challenge is that you involve many more actors than we traditionally do in the automotive industry. You involve a service provider, which also has requirements on the chain of functionality. The value chain for the customer, I mean. Suddenly, you are outside the system 'car' to validate that you provide the right quality and services.

While the digitization of the automotive industry has led to increasingly complex business processes, the influence of outside actors on the user value produced was new to the organization. Indeed, this outside influence over user value was perceived extra problematic because of significant differences between the automotive and consumer electronics industries. Reflecting on one such difference, the same infotainment manager expressed:

Well, since we have a development cycle of, maybe, two or three years and they have a cycle of one year, or even six months, as well as a product life time that is much shorter than our 10 years. The two industries do not map each other at all.

The R\&D people predicted that the 'life cycle issue' would result in difficulties for Saab to keep pace enough to support new connecting devices and emerging use patterns over time. In particular, speeding up the development process was considered as a risky strategy that eventually would undermine other types of user expectations and value. A manager responsible for the R\&D projects on infotainment explained:

It takes up to four years to develop a product and this process is associated with endless testing, development hours, and this is due to the fact that customers do not accept rebooting the car every day. The car is supposed to be up-and-running in at least fifteen years without any significant maintenance.

In this situation, Saab figured that they were in need of an organizational capability that would cater for continuous user value satisfaction. Saab employees envisioned various new approaches for securing user value, including innovative forms of organization and flexible in-car software platforms:

I think a new type of organization is needed that take care of this. It must be more oriented towards consumer electronics (. . .). It should be focused on shortening cycles...

We must make it possible to handle updates. I also believe that it would be useful to open a part of the in-car platform to 3rd parties or even end-users to enable software download for upgrading the platform so that it supports their devices. 
These reflections are indicative of the fact that Saab recognized the intricate relationship between new connecting devices and new use cases and user expectations as a key challenge in leveraging user value of MTIS. As the testimonies highlighted, adaptation of the organization's development practices was seen as necessary to secure user value over time.

\section{IMPLICATIONS}

Over the years, user involvement has been an important theme in IS research. However, it has been noted that the theoretical progress within the area has been relatively stalled, thus inspiring researchers to reconceptualize received theory to better cater for some of the profound changes that IS practice has undergone (Markus \& Mao, 2004). Conducting such a reconceptualization on the basis of the classical relationship between user involvement and system success (see e.g. McKeen et al., 1994), Markus \& Mao (2004) identify gaps or logical inconsistencies in extant theory and formulate a number of propositions useful for guiding future research in the area. Given the need for a refined theory explaining how and why user involvement affects system success, studies that explore new contingency factors determining the relative usefulness of user involvement are needed.

Technological innovations that contribute to increased system complexity and stakeholder ambiguity are one such contingency factor. For instance, because systems are increasingly interconnected by means of network technologies, resulting information environments consisting of loosely connected systems increase the number of designers, user collectives and other stakeholders over time. While such system complexity and stakeholder ambiguity partly changes the user involvement landscape, however, received theory tends to overlook implications of such changes in the underlying technology. Indeed, analysis of technological changes would usefully complement an updated view on user involvement for today's changing IS contexts. We believe that our exploration of user involvement in developing a MTIS may provide such a complement along the lines of Markus and Mao's redefined portrait of stakeholder participation.

The SeamlessTalk case exemplifies a systems development process where user involvement was a key element. In commenting the key lesson learned in the project, an infotainment manager at Saab noted:

We had the chance to look closely at user value for the customer in everyday life, between office, car, and home. [We had] a very user-focused looking at how to simplify the transitions between contexts. [. . .] We basically learned that a Saab customer has a life outside the car too.

The project can be considered successful in that the system concept appeared in production cars a few years later. Despite the successful outcome, however, there were things that could have been done differently. Reflecting on our case, it can be noted that the nature of MTIS affects user involvement practices. In substantiating this argument, the paper develops implications for how user involvement may be adapted to the development of MTIS. 


\section{Use context switching}

The switching contexts feature of MTIS is at odds with the assumption of non-switching contexts reflected in the user involvement literature. In our study, we noted how one source of user requirements on NDI solutions was related to use patterns being enacted across multiple contexts, i.e, boundary-spanning use patterns. For instance, the SeamlessTalk prototype did not synchronize the recent number list. However, the evaluation showed that this boundaryspanning call initiation use pattern was perceived important. Even though the in-car user interface was radically different, this use pattern was deemed relevant in the car setting as well. In other words, when approaching new settings, it seems that users tend to stick to use patterns already established in other settings. On a general level, this insight implies that the multi-contextuality of MTIS requires a new angle on context interpretation where all involved contexts, and their interactions, are considered.

In developing MTIS, attention must be paid to the specifics of each of the local contexts. Such analysis can be usefully accomplished by using ethnography, group elicitation techniques, usability engineers or other established means of capturing user requirements. In addition, however, attention must be paid to the intersecting use patterns of these contexts. Traditional user-centred techniques such as ethnography, primarily embracing local work processes and settings, are somewhat inadequate for capturing use patterns that span contexts. Analysis of such intersections is likely to present ambiguities, because requirements of each context may not be fully mitigated. For instance, convenience-oriented requirements on the user interface of media players on cellphones may not apply to the safety-oriented design guidelines used in the in-car user interface design of media players.

In sum, in developing MTIS, it is important to (a) identify and analyse the contexts within the realm of the included systems, and (b) identify and analyse users' boundary-spanning use patterns by conducting comparative analysis of these contexts as to address the switching context feature of MTIS. These two steps are relevant to enable mitigation of the possible contradictions between requirements of the different local contexts.

\section{Temporary system relationships}

MTIS are characterized by temporary relationships between heterogeneous systems. Even though the extant user involvement literature implies interconnected systems (e.g. a traditional management information system implies an operational transaction system ${ }^{1}$ ), it does not consider the implications of either temporary relationships or heterogeneity between systems. Rather than adhering to a paradigm of permanent relationship between homogenous systems, MTIS deal with temporary connections to technologies following other innovation trajectories and design guidelines. As a result, MTIS cannot be developed in isolation from the emerging information services, which they are intended to support. MTIS need to serve such services over time to sustain user value.

${ }^{1}$ We are grateful to the special issue editors for providing this example. 
Given the universal character of information services supported by NDI solutions, the problem of unknown users is fairly analogue to the case of packaged software development. In the SeamlessTalk case, it was difficult to identify users, because product development settings seldom involve people with clear-cut stakes. Thus, we had no choice but concentrate on surrogate users, i.e. users who were likely to be future NDI users. The fact that we selected surrogate users in the requirements determination phase and participative users in the evaluation phase did not help us resolve some of the major hurdles in realizing useful NDI systems. This was related to the temporary relationships between heterogeneous systems aspect of MTIS, which differ from most packaged software situations.

In looking back on the project, Saab employees noted that the temporary relationship between heterogeneous systems was not really addressed by the user involvement process throughout the systems development project. In the NDI case, systems are supposed to support most, if not all, mobile devices over a number of different information services. This means that the ultimate test of an NDI system's capacity to produce user value is over time, because the system is supposed to support ubiquity of information services running on future devices. SeamlessTalk only supported two Nokia phones, which we provided to the users. They became outdated long before the system was intended to be implemented in production cars.

This lesson learned made Saab employees reflect upon time cycle differences between the automotive and consumer electronics industries, and, in particular, on the consequences for sustaining user value over time. Clearly, user involvement in the development phase is not enough when dealing with temporarily interconnected systems. In a post-adoption phase, further development of the system is needed. Such a phase would benefit from direct links with users and third party organizations. It is therefore imperative to develop a software organization that encourage active user involvement and draw on continuous user feedback. Looking at the literature, community-based development of software has become immensely popular for sustaining and improving user value over time (see e.g. Lee \& Cole, 2003; Holmström \& Henfridsson, 2006). Internet technologies are used to handle improvements of the software ranging from bug-fixes to new functionality by a user collective that co-produces the system.

While open source software development draw on a relatively homogeneous knowledge base (Yoo et al., 2008), however, MTIS designers must handle the fact that systems are interconnected but heterogeneous. Typically, control can only be exercised over one subsystem. In order to handle this heterogeneity and the time cycle differences, Saab employees speculated about community use where an in-car open software architecture would facilitate system updates over time, ensuring continuous adaptation with regard to the rapid changes in consumer electronics with new connecting devices and use cases launched frequently. Heterogeneity would still remain in terms of fundamental differences in, e.g. user interface requirements, but, on a software platform level, such a strategy would help balancing the heterogeneity of MTIS.

In sum, in developing MTIS, it is important to (a) view user involvement as a continuous activity that extends into a post-adoption phase, (b) design an open software architecture that allows flexible updates as new temporary relationships with connecting devices need to be 
supported, and (c) develop community management capabilities that help tracing emerging information services for securing implementation of new features that serve users' changing use patterns.

\section{CONCLUSION}

The implications derived from this research indicate that we are witnessing a slight drift from the traditional way of conceptualizing development practices in general and user involvement in particular. This drift may be interpreted in at least two ways. One interpretation is that there is a need for more studies examining how existing practices for user involvement can be adapted to cater for the reality of MTIS development. An alternative interpretation is that the notion of user involvement as we know it is at odds with the emerging new landscape of systems development.

Regardless of which interpretation is correct, our study has captured one instantiation of the increasingly complex interplay between heterogeneous actors and technologies in attempts to develop usable MTIS. We expect to see a lot more future work in this area. In particular, more studies are needed about the nature of approaches to user involvement that help designers avoiding the pitfall of underestimating development challenges stemming from boundaryspanning usages of connected technologies. Indeed, the design of MTIS is about dwelling with new use cases and user expectations being continuously created by ever-emerging devices and services.

\section{ACKNOWLEDGEMENTS}

VINNOVA and the participating organizations funded this work. We are also deeply indebted to the industry participants of our project. Special thanks to Helén Falkås at Saab Automobile. Thanks are also due to the special issue editors, Juhani livari, Hannakaisa Isomäki, Samuli Pekkola and two anonymous reviewers for their useful comments.

\section{REFERENCES}

Abowd, G.D. \& Mynatt, E.D. (2000) Charting past, present, and future research in ubiquitous computing. ACM Transactions on Computer-Human Interaction, 7, 29-58.

Adman, P. \& Warren, L. (2000) Participatory sociotechnical design of organizations and information systems an adaptation of ETHICS methodology. Journal of Information Technology, 15, 39-51.

Andersson, M. \& Lindgren, R. (2005) The mobilestationary divide in ubiquitous computing environments: lessons from the transport industry. Information Systems Management, 22, 65-79.

Andersson, M., Lindgren, R. \& Henfridsson, O. (2008) Architectural knowledge in inter-organizational IT innovation. Journal of Strategic Information Systems, 17, 19-38.

Barki, H. \& Hartwick, J. (1989) Rethinking the concept of user involvement. MIS Quarterly, 13, 53-63.

Baskerville, R. \& Pries-Heje, J. (1999) Grounded action research: a method for understanding IT in practice. 
Accounting, Management \& Information Technologies, 9, 1-23.

Beath, C.M. \& Orlikowski, W.J. (1994) The contradictory structure of systems development methodologies: deconstructing the IS-user relationship in information engineering. Information Systems Research, 5, 350377.

Beyer, H. \& Holtzblatt, K. (1995) Apprenticing with the customer. Communications of the ACM, 38, 45-52.

Beyer, H. \& Holtzblatt, K. (1999) Contextual design. Interactions, 6, 32-42.

Broy, M., Krüger, I.H., Pretschner, A. \& Salzmann, C. (2007) Engineering automotive software. Proceedings of the IEEE, 95, 356-373.

Cole, R., Purao, S., Rossi, M. \& Sein, M.K. (2005) Being proactive: where action research meets design research. In: Proceedings of ICIS26, King, W.R. \& Torkzadeh, R. (eds), pp. 325-336. Las Vegas, NV, USA. Cooper, C. \& Bowers, J. (1997) Representing the users: notes on the disciplinary rhetoric of human-computer interaction. In: The Social and Interactional Dimensions of Human-Computer Interfaces, Thomas, P.J. (ed.), pp. 48-66. Cambridge University Press, Cambridge, UK.

Cooper, R.B. (2000) Information technology development creativity: a case study of attempted radical change. MIS Quarterly, 24, 245-276.

Damodaran, L. (1996) User involvement in the system design process - a practical guide for users. Behaviour \& Information Technology, 15, 363-377.

Davison, R.M., Martinsons, M.G. \& Kock, N. (2004) Principles of canonical action research. Information Systems Journal, 14, 65-86.

Dey, A.K., Abowd, G.D. \& Salber, D. (2001) A conceptual framework and a toolkit for supporting the rapid prototyping of context-aware applications. Human-Computer Interaction, 16, 97-166.

Ehn, P. (1988) Work-Oriented Design of Computer Artifacts. Arbetslivscentrum and Almqvist \& Wiksell International, Stockholm, Sweden.

Ehn, P. (1993) Scandinavian design: on participation and skill. In: Participatory Design: Principles and Practices, Schuler, D. \& Namioka, A. (eds), pp. 41-77. Lawrence Erlbaum, Hillsdale, NJ, USA.

Fitzgerald, B. (2006) The transformation of open source software. MIS Quarterly, 30, 587-598.

Gallivan, M.J. \& Keil, M. (2003) The user-developer communication process: a critical case study. Information Systems Journal, 13, 37-68.
Gould, J.D. \& Lewis, C. (1985) Designing for usability: key principles and what designers think. Communications of the ACM, 28, 300-311.

Greenbaum, J. \& Kyng, M. (1991) Design at Work: Cooperative Design of Computer Systems. Erlbaum, Hillsdale, NJ, USA.

Grudin, J. (1991) Interactive systems: bridging the gaps between developers and users. IEEE Computer, 24, 59-69.

Hansson, C., Dittrich, Y. \& Randall, D. (2006) How to include users in the development of off-the-shelf software: a case for complementing participatory design with agile development. In: Proceedings of HICSS-39, Sprague, Jr., R.H. (ed.) [CD-ROM]. Big Island, HI, USA. IEEE Press, Washington, DC, USA.

Hawk, S.R. \& Dos Santos, B.L. (1991) Successful system development: the effect of situational factors on alternate user roles. IEEE Transactions on Engineering Management, 38, 316-327.

Henfridsson, O. \& Lindgren, R. (2005) Multi-contextuality in ubiquitous computing: investigating the car case through action research. Information and Organization, 15, 95-124.

Hirschheim, R. \& Klein, H.K. (1994) Realizing emanicipatory principles in information systems development: the case for ETHICS. MIS Quarterly, 18, 83-109.

Holmström, H. \& Henfridsson, O. (2006) Improving packaged software through online community knowledge. Scandinavian Journal of Information Systems, 18, 3-36.

Howcraft, D. \& Wilson, M. (2003) Paradoxes of participatory practices: the Janus role of the systems developer. Information and Organization, 13, 1-24.

Hughes, J., Randall, D. \& Shapiro, D. (1992) Faltering from ethnography to design. In: Proceedings of CSCW '92, Turner, J. \& Kraut, R. (eds), pp. 115-122. Toronto, ON Canada. ACM Press, New York, USA.

livari, J. \& livari, N. (2006). Varieties of user-centeredness. In: Proceedings of HICSS-39, Sprague, Jr., R.H. (ed.). Big Island, HI, USA. IEEE Press, Washington, DC, USA.

livari, J. \& Lyytinen, K. (1998) Research on information systems development in Scandinavia - unity in plurality. Scandinavian Journal of Information Systems, 10, 135186.

livari, N. (2006) 'Representing the user' in software development - a cultural analysis of usability work in the product development context. Interacting with Computers, 18, 635-664. 
Keil, M. \& Carmel, E. (1995) Customer-developer links in software development. Communications of the ACM, 38, 33-44.

Klein, H.K. \& Myers, M.D. (1999) A set of principles for conducting and evaluating interpretive field studies in information systems. MIS Quarterly, 23, 67-93.

Kleinrock, L. (2001) Breaking loose. Communications of the ACM, 44, 41-45.

Kujala, S. (2003) User involvement: a review of the benefits and challenges. Behaviour \& Information Technology, 22, 1-16.

Kyng, M. \& Mathiassen, L. (1982) Systems development and trade union activities. In: Information Society, for Richer, for Poorer, Bjorn-Andersen, N. (ed.), pp. 247260. North-Holland, Amsterdam, the Netherlands.

Lee, G.K. \& Cole, R.E. (2003) From a firm-based to a community-based model of knowledge-creation: the case of the Linux kernel development. Organization Science, 14, 633-649.

Lindgren, R., Henfridsson, O. \& Schultze, U. (2004) Design principles for competence management systems: a synthesis of an action research study. MIS Quarterly, 28, 435-472.

Lindgren, R., Andersson, M. \& Henfridsson, O. (2008) Multi-contextuality in boundary-spanning practices. Information Systems Journal, 18, 641-661.

Lyytinen, K. \& Rose, G.M. (2003) The disruptive nature of information technology innovations: the case of internet computing in systems development organizations. MIS Quarterly, 27, 557-595.

Lyytinen, K. \& Yoo, Y. (2002a) Issues and challenges in ubiquitous computing. Communications of the ACM, 45, 63-65.

Lyytinen, K. \& Yoo, Y. (2002b) Research commentary: the next wave of nomadic computing. Information Systems Research, 13, 377-388.

McKeen, J.D., Guimaraes, T. \& Wetherbe, J.C. (1994) The relationship between user participation and user satisfaction: an investigation of four contingency factors. MIS Quarterly, 18, 427-451.

March, S., Hevner, A. \& Ram, S. (2000) Research commentary: an agenda for information technology research in heterogeneous and distributed environments. Information Systems Research, 11, 327341.

Markus, M.L. \& Mao, J.-Y. (2004) Participation in development and implementation - updating an old, tired concept for today's IS contexts. Journal of the Association for Information Systems, 5, 514-544.
Muller, M.J., Hallewell Haslwanter, J. \& Dayton, T. (1997) Participatory practices in the software lifecycle. In: Handbook of Human-Computer Interaction, Helander, M., Landauer, T.K. \& Prabhu, P (eds), pp. 255-297. Elsevier, Amsterdam, the Netherlands.

Mumford, E. (1983) Designing Human Systems for New Technology: The ETHICS Method. Manchester Business School, Manchester, UK.

Mumford, E. (1993) The participation of users in systems design: an account of the origin, evolution, and use of the ETHICS method. In: Participatory Design: Principles and Practices, Schuler, D. \& Namioka, A. (eds), pp. 257-270. Erlbaum, Hillsdale, NJ, USA.

Newman, M. \& Noble, F. (1990) User involvement as an interaction process: a case study. Information Systems Research, 1, 89-113.

Sabherwal, R. \& Elam, J. (1995) Overcoming the problems in information systems development by building and sustaining commitment. Accounting, Management \& Information Technologies 5, 283-309.

Sawyer, S. (2000) Packaged software: implications of the differences from custom approaches to software development. European Journal of Information Systems, 9, 47-58.

Tuunanen, T. (2003) A new perspective on requirements elicitation methods. Journal of Information Technology Theory and Application, 5, 45-72.

Weiser, M. (1991) The computer for the 21st century. Scientific American, 265, 94-104.

Wilson, A., Bekker, M., Johnson, P. \& Johnson, H. (1997) Helping and hindering user involvement - a tale of everyday design. In: Conference on Human Factors in Computing Systems (CHI), Pemberton, S. (ed.), pp. 178-185. Atlanta, GA, USA. ACM Press, New York, USA.

Wixon, D. \& Wilson, C. (1997) The usability engineering framework for product design and evaluation. In: Handbook of Human-Computer Interaction, Helander, M., Landauer, T.K. \& Prabhu, P (eds), pp. 653-688. Elsevier, Amsterdam, the Netherlands.

Yoo, Y., Lyytinen, K. \& Boland, R.J. (2008) Distributed innovation in classes of networks. In: Proceedings of HICSS-41, Sprague, Jr., R.H. (ed.). Waikoloa, Big Island, HI, USA. IEEE Press, Washington DC, USA.

\section{Biographies}

Ola Henfridsson is the Research Manager of the Automotive Group at the Viktoria Institute, Göteborg, Sweden. 
$\mathrm{He}$ is also a Professor of Informatics at Jönköping International Business School, Jönköping University, Sweden. His research interests include IT innovation, IT and organizational change, as well as action and design research. The outcome of this research has been published in Information and Organization, Information Systems Journal, IT and People, Journal of Strategic Information Systems, MIS Quarterly and other journals in the information systems discipline. Ola serves on the editorial boards of the MIS Quarterly, Information Technology and People and Journal of the Association for Information Systems. He is also a Region 2 representative on the Association for Information Systems (AIS) Council.
Rikard Lindgren is a Professor of Informatics at the University of Gothenburg, Sweden. He is also managing the Transport Research Group at the Viktoria Institute. His research has been published in European Journal of Information Systems, Information and Organization, Information Systems Journal, Journal of Strategic Information Systems, MIS Quarterly and other journals in the information systems discipline. Currently, Professor Lindgren serves on the editorial boards of Information Systems Research, Journal of Information \& Knowledge Management and Scandinavian Journal of Information Systems. 